\title{
A comparison of simple analytical methods for determination of fluoride in microlitre-volume plasma samples
}

FV Zohoori ${ }^{1}$, A Maguire ${ }^{2}$, EA Martinez-Mier ${ }^{3}$, M Buzalaf $^{4}$, R Sanderson ${ }^{5}$, GJ Eckert ${ }^{6}$,

${ }^{1}$ School of Health and Social Care, Teesside University, UK

${ }^{2}$ Centre for Oral Health Research (COHR), School of Dental Sciences, Newcastle University, UK

${ }^{3}$ Operative Dentistry and Dental Public Health, Indiana University School of Dentistry, US

${ }^{4}$ Bauru School of Dentistry, University of São Paulo, Brazil

${ }^{5}$ Evidence \& Policy Research Group, School of Natural \& Environmental Science, Newcastle University, UK

${ }^{6}$ Biostatistician Supervisor in Biostatistics, School of Medicine, Indiana University, US

Short title: Determination of fluoride in microlitre-volume plasma

Key words: Fluoride, plasma, method, microlitre-volume

Corresponding author:

Professor FatemehVida Zohoori;

Health and Social Care Institute

Teesside University

Middlesbrough, TS1 3BA

UK

Declaration of interests

The study was funded by a grant from The Borrow Foundation. The authors report no conflicts of interest. The views expressed in this paper are those of the authors and not those of the funding body.

Acknowledgement:

The authors would like to thank N Omid, I Oladipo, L Grizzo, C Buckley, P Chandrappa and GA Castiblanco Rubio for their technical supports. Support from The Borrow Foundation is gratefully acknowledged. 


\section{$\underline{\text { Abstract: }}$}

The aim was to compare potential methods for fluoride analysis in microlitre-volume plasma samples containing nano-gram amounts of fluoride. Methods: A group of four laboratories analysed a set of standardised biological samples as well as plasma to determine fluoride concentration using three methods. In Phase-1, fluoride analysis was carried out using the established HMDS-diffusion method (1 ml-aliquot/analysis) to obtain preliminary measurement of agreement between the laboratories. In Phase-2, the laboratories analysed the same samples using a micro-diffusion method and knownaddition technique with $200 \mu 1$-aliquot/analysis. Coefficients of Variation (CVs) and intra-class correlation coefficients (ICCs) were estimated using ANOVA to evaluate the amount of variation within- and between-laboratories. Based on the results of the Phase-2 analysis, 20 human plasma samples were analysed and compared using the HMDS-diffusion method and known-addition technique in Phase-3. Results: Comparison of Phase-1 results showed no statistically significant difference among the laboratories for the overall data set. The mean between- and within-laboratory CVs and ICCs were $<0.13$ and $\geq 0.99$, respectively, indicating very low variability and excellent reliability. In Phase-2, the overall results for between-laboratory variability showed a poor CV (1.16) and ICC (0.44) for the micro-diffusion method, whereas with the known-addition technique the corresponding values were 0.49 and 0.83 . Phase- 3 results showed no statistically significant difference in fluoride concentrations of the plasma samples measured with HMDS-diffusion method and known-addition technique, with a mean (SE) difference of 0.002(0.003) $\mu \mathrm{g} / \mathrm{ml}$. In conclusion, the known-addition technique could be a suitable alternative for measurement of fluoride in plasma with microlitre-volume samples. 


\section{Introduction:}

It has been well established that topical fluoride $(\mathrm{F})$ has an important role in the prevention of dental caries. However, excessive systemic ingestion of F during enamel development prior to tooth eruption can increase the risk of development of dental fluorosis. Therefore, it is important to assess $\mathrm{F}$ exposure and retention in very young children on a regular basis using a suitable biomarker for $\mathrm{F}$. The World Health Organisation (WHO) has stated that "a fluoride biomarker is of value primarily for identifying and monitoring deficient or excessive intakes of biologically available fluoride" [World Health Organization, 1994]. For a biomarker to be suitable, it needs to be valid, reliable and practical in terms of collection and analysis with the practical aspects of sample collection being especially pertinent in young children.

Following ingestion of $\mathrm{F}$, the plasma $\mathrm{F}$ concentration increases rapidly, reaching its peak within 20-60 minutes. Since the rise in plasma F concentration is proportional to $\mathrm{F}$ intake, plasma has been suggested as a reliable indicator of $\mathrm{F}$ exposure in humans. Additionally, from a pharmacokinetic perspective, plasma is considered as the 'central compartment' of F distribution systemically, since it is the fluid that F must pass into and then from, to be distributed around and eliminated from, the body [Whitford, 1996]. Despite the importance of plasma F for pharmacokinetic and analytical epidemiology studies, there are insufficient data on plasma F concentrations, particularly in children, to determine normal baseline values for individuals living in fluoridated and non-fluoridated communities with different degrees of $F$ intake and exposure [Rugg-Gunn et al., 2011]. Pharmacokinetic and analytical epidemiology studies usually involve venous blood sampling by direct puncture to a vein, most often located in the antecubital area of the arm or the dorsal aspect (top) of the hand. In surveys involving measurements of several analytes in blood, the relatively large volumes of blood required may also be an insurmountable limitation. In addition, venous blood sampling is deemed ethically unacceptable or impractical in most studies with healthy young children. As a result, capillary blood is often the method of choice for infants, very young children, and elderly patients with fragile veins, and is collected by dermal puncture of fingertip or heel.

Determination of $\mathrm{F}$ in biological samples comprises several steps which may include pre-treatment of samples, separation and concentration of $\mathrm{F}$, actual measurement of $\mathrm{F}$ ions, calculation of final concentration per unit of sample, and presentation of the data [Venkateswarlu, 1990]. The most accurate sample preparation technique uses a diffusion method, such as acid-hexamethyldisiloxane (HMDS) diffusion [Taves, 1968] to separate F ion from the interfering substances in the sample and transfer it to a trapping solution of small volume to increase the final $\mathrm{F}$ concentration in the solution. The most frequently employed method for measurement of $\mathrm{F}$ involves the use of ion-selective electrode (ISE)-based potentiometric methods which measure free $\mathrm{F}$ ions in aqueous solutions fairly 
quickly. Blood plasma contains two general forms of F: ionic (inorganic) and non-ionic (organic). The ionic form is the one of interest in dentistry, medicine, and public health [Whitford, 1996] which can be principally determined by F-ISE.

To optimise the quality of dental research involving $\mathrm{F}$ assay and minimise between-laboratories $\mathrm{F}$ analysis inconsistencies, standardised $\mathrm{F}$ analysis techniques have been developed and tested based on a HMDS-diffusion method using F-ISE [Martínez-Mier et al., 2011]. The techniques are used increasingly widely by laboratories undertaking $\mathrm{F}$ analysis of biological and non-biological samples as they meet requirements for accuracy and reliability at relatively low-cost.

In adult humans, plasma $\mathrm{F}$ concentration might be as low as 6.8ng/ml [Buzalaf, 2011]. Considering the limit of sensitivity of the F-ISE, which is about $\left.10^{-6} \mathrm{~mol} / 1 \mathrm{l}=0.02 \mu \mathrm{g} / \mathrm{ml}=20 \mathrm{ng} / \mathrm{ml}\right)$ [Whitford, 1996], a minimum volume of $1000 \mu 1$ of plasma is required to ensure acceptable $F$ measurement in duplicate $(500 \mu \mathrm{l}$ per replicate) using the gold standard HMDS diffusion method in which the $\mathrm{F}$ is concentrated in a 75-100 $\mu 1$ volume prior to analysis [Taves, 1968; Martínez-Mier et al., 2011]. The volume of plasma that can be obtained from a unit of whole blood is determined by the volume of blood collected and the donor percent haematocrit $(\% \mathrm{Hct})$. The theoretical range of plasma volume that can be recovered from a unit of whole blood is 40-60\% [PALL Medical, 2010]. Therefore, a minimum of approximately $2 \mathrm{ml}$ blood is needed for duplicate (or $3 \mathrm{ml}$ for triplicate) plasma $\mathrm{F}$ analysis using the gold standard HMDS-diffusion method [Martínez-Mier et al., 2011].

Due to the importance of F research in dentistry, several F-ISE-based methodologies for sample manipulation and $\mathrm{F}$ analysis on very small sample volumes have been explored for measuring $\mathrm{F}$ in biological samples such as saliva, plasma and dental plaque (biofilm). Several techniques, including micropipette procedures for transferring samples, preparation of micro F-ISE, and methods for adapting standard electrodes have been developed for various biological samples containing nano- or sub-nano-gram amounts of F [Hallsworth et al., 1976; Vogel et al., 1990]. Despite promising results with these methods for samples such as saliva, dental plaque, and tooth enamel, they have not been widely employed due to their cost and time-consumption in addition to the need for careful attention to technique which requires extensive experience.

Using the relatively straightforward known-addition technique, Ekstrand [Ekstrand, 1977] reported a satisfactory reproducibility with $150 \mu$ l plasma samples containing $\geq 20 \mathrm{ng} F$. A rather simple microdiffusion technique, based on the gold standard HMDS-diffusion method, has also been developed for dental plaque [Martinez-Mier et al., 2010] which can easily be adapted for samples of plasma. Therefore, the aim of this study was to investigate the precision and accuracy of these two simple methods for $\mathrm{F}$ analysis in micro-litre volume plasma samples containing nano- or sub-nano-gram amounts of F, in comparison with the gold standard HMDS-diffusion method. 


\section{Material and methods}

Four internationally recognised laboratories with track record expertise in $\mathrm{F}$ analysis participated in this study. The sites comprised University of São Paulo (Brazil), Newcastle University (UK), Teesside University (UK), and Indiana University (US). Each collaborative laboratory received a set of samples comprising: five F standards as Reference Materials (RMs), four samples of National Institute of Standards and Technology (NIST) traceable F standards, two samples of Urine Certified Reference Fluoride (UCRF), and three samples of freeze-dried powdered plasma samples.

The five RMs with F concentrations of 0.01, 0.02, 0.06, 0.2 and $1.0 \mathrm{ppm}$ were prepared from a certified 100 ppm F standard (940907, Fisher Scientific) in one of the centres. The four NIST traceable standards were also prepared from a NIST-certified $10 \mathrm{ppm}$ F solution at another centre. Samples of UCRF (PC-U-F1703) were purchased from 'Laboratoire de santé publique du Québec' (QC H9X 3R5) and powdered plasma (P9523) from Sigma-Aldrich (Saint Louis, MO 63103, USA) by one centre. All the samples were prepared and/or purchased by staff who were not directly involved in sample analysis and labelled with a code. The blind-labelled samples were then distributed among the four collaborative laboratories. No specific preparation was used for RMs, NIST traceable standards or UCRFs in each laboratory. However, each vial of dried plasma was reconstituted with $5 \mathrm{ml}$ deionised distilled water (DDW) to produce $5 \mathrm{ml}$ liquid plasma. All samples were stored in a freezer $\left(-20^{\circ} \mathrm{C}\right)$ until $\mathrm{F}$ analysis.

The study was then undertaken in three phases. In Phase 1, a between-laboratory comparison of the gold HMDS-diffusion method was conducted. The four collaborative laboratories analysed a set of samples $(\mathrm{n}=14)$, in triplicate ( $1 \mathrm{ml}$ sample per replicate), in order to obtain a preliminary measure of agreement between the laboratories. Within each laboratory, the samples were re-analysed on a separate day to obtain the within-laboratory agreement/reliability. In Phase 2, all four laboratories analysed the same set of samples as Phase 1 but in a lower volume (200 $\mu \mathrm{l})$ using the micro-diffusion method as well as known-addition technique. Detailed information regarding the three analytical methods (HMDS-diffusion method, micro-diffusion method and known-addition technique) is available as online supplementary materials on the Karger website.

The results from the tests in Phases 1 and 2 were distributed to all laboratories and a review involving comparison of the three methods for $\mathrm{F}$ analysis was conducted by email and at a face-face meeting. According to the results, the choice of methods for Phase 3 were agreed among the laboratories.

In Phase 3, the selected methods were used to analyse 20 human plasma samples by one laboratory.

\section{Statistical analysis}


i) Sample size: The sample size calculation was based on the precision of the estimate of the withinand between- laboratory intra-class correlation coefficients (ICCs). The samples were measured twice at each of the four laboratories. With a sample size of $n=14$ samples, the $95 \%$ lower confidence bound for the ICCs would extend, at most, 0.10 from the estimated ICCs, assuming the within-laboratory ICCs were at least 0.90 and the between-laboratory ICCs were at least 0.75 .

ii) Statistical analysis: Plasma samples for which the F concentration was not certified, F standards and NIST traceable standards were evaluated for the amount of variation within- and betweenlaboratories using random effects analysis of variance models (ANOVAs). The ANOVAs also provided estimates of the Coefficients of Variation (CVs) and intra-class correlation coefficients (ICCs). Pairwise comparisons among the laboratories were performed using a Tukey-Kramer adjustment for multiple comparisons. Analyses were performed using SAS version 9.3 (SAS Institute Inc., Cary, NC).

Trueness and precision testing of the agreed-upon methods were evaluated using the ISO Guide 32: Calibration in analytical chemistry and use of certified reference materials [1997] and the NIST Special Publication 829: Use of NIST traceable standards for decision on performance of analytical chemical methods and laboratories [Becker D et al., 1992]. Trueness and between-laboratory precision were based on confidence intervals that use both the within- and between-laboratory error terms. The trueness and precision analyses included the plasma samples in addition to the F standards and NIST traceable standards. The F concentrations from the Phase $1 \mathrm{~F}$ analysis of the plasma samples were considered as the reference values for comparison with the lower volume sample $\mathrm{F}$ analyses.

Linear mixed effects models [Pinheiro and Bates, 2000] were used to determine the effects of assay method on the measured F concentration. Method, laboratory and true F concentration (i.e. actual expected known value) were used as fixed effects, whilst phase and day were treated as nested random effects. Separate mixed-effects intercept-only analyses were also undertaken on the difference between the measured and true F concentrations (ideally zero), with phase, laboratory and method as nested random effects. Quality of the fitted models was assessed by quantile-quantile plots (QQ-plot), residuals and predicted vs observed values. Analyses were undertaken in R using the nlme library [Pinheiro J, 2017].

\section{Results}

Table 1 presents the overall between- and within-laboratory variabilities in the F concertation of samples collectively, by method, when the results from all laboratories were pooled together. Overall, results for between-laboratory variability showed a poor ICC of 0.44 for the micro-diffusion method 
but excellent ICCs for the HMDS-diffusion method (0.99) and the known-addition technique (0.83). There was also a poor $\mathrm{CV}$ of 1.162 for the micro-diffusion method, however, the between-laboratory variability showed an ICC of more than 0.75 (a cut-off point for an excellent agreement) for the three methods.

When looking at the overall data set, the comparison of the mean F concentration of all samples showed no statistically significant difference among the four laboratories when the samples were analysed by the HMDS-diffusion method as well as with the known-addition technique. However, the between-laboratory comparison of the mean F concentrations of all samples, measured by the microdiffusion method, found a statistically significantly $(\mathrm{p}=0.05)$ difference in the measured $\mathrm{F}$ concentrations between Laboratory 1 and 3.

The within-laboratory variation in the overall measured $\mathrm{F}$ concertation by method is presented in Table 2. The mean CVs for the laboratories were below 0.1, and the ICCs were above 0.96 with the gold standard HMDS-diffusion method and the known-addition technique, whereas the ICC was as low as 0.37 and $\mathrm{CV}$ as high as 0.671 with the micro-diffusion method.

Comparison of the results for each individual sample among laboratories indicated statistically significant differences among the four laboratories for some samples (Table 3). For the HMDSdiffusion method, Laboratory 2 had statistically significantly ( $\mathrm{p}=0.04$ ) lower F measurements than Laboratory 4 for only one sample (RM-C, $0.06 \mathrm{ppmF}$ ) with a mean (SE) difference of $-0.028(0.002)$ $\mu \mathrm{g} / \mathrm{ml}$. The between-laboratory comparison of the results obtained by the micro-diffusion method and known-addition technique revealed statistically significant differences obtained for certain samples (e.g. NIST-A, NIST-D, NIST-C, URCF-2) among some laboratories as demonstrated in Table 3.

The true F concentrations of all samples, apart from plasma, were known. Therefore, for each individual sample (apart from plasma), its measured $\mathrm{F}$ concentration by each of the four laboratories for each method were pooled and compared with its true (expected known) value (Figure 1). Table 4 shows the results of the mixed-model analysis, comparing the measured $\mathrm{F}$ concentrations with the expected F concentration values, with laboratory and method as fixed effects and phase and day as nested random effects. The results showed a strong relationship between measured and true (expected known) F concentration values. However, there was a significant laboratory effect, with Laboratory 3 noticeably different from the other 3 laboratories. The comparison of the measured $\mathrm{F}$ concentration of the three low F concentration samples (i.e. $0.01,0.02$ and $0.06 \mu \mathrm{g} / \mathrm{ml}$ ) with predicted values from the fitted model, across all the laboratories, showed that the predicted F concentrations by the knownaddition were much closer to the values predicted by the established HMDS-diffusion method. The QQ-plots for the HMDS-diffusion method and known- addition technique gave approximately straight lines (Figure 2), confirming the validity of the statistical models used in the analysis. 
Mean (SE) F concentrations of the 20 plasma samples which were measured by the established gold standard method (i.e. HMDS-diffusion) and the known-addition technique were $0.040(0.006)$ and $0.038(0.006) \mu \mathrm{g} / \mathrm{ml}$, respectively. The mean (SE) differences in F concentration between these two methods was $0.002(0.003) \mu \mathrm{g} / \mathrm{ml}$, with a $95 \% \mathrm{CI}$ of $-0.005,+0.009 \mu \mathrm{g} / \mathrm{ml}$; indicating a nonstatistically significant difference in the measured $\mathrm{F}$ concentrations between the two methods.

\section{Discussion}

Pharmacokinetic and analytical epidemiology studies, particularly in dentistry, often seek to assess F concentrations in very small volume plasma samples. In the current literature, there is very limited information available on the reliability of quantitative measures of $\mathrm{F}$ in micro-litre volume plasma samples containing nano- or sub-nano-gram amounts of $\mathrm{F}$. The current study quantified the reliability of the measurement of $\mathrm{F}$ in micro-litre volumes of plasma using micro-diffusion and known-addition techniques in comparison with the gold standard HMDS-diffusion method.

The main purpose of carrying out $\mathrm{F}$ analysis of the samples using the established gold standard HMDS-diffusion method with a minimum sample volume of $1 \mathrm{ml}$ per replicate, in the current study, was to; i) obtain a preliminary measure of agreement between the laboratories in determining $\mathrm{F}$ concentration of samples, and; ii) use as a gold standard method for assessment of the other two methods. The comparison of the results obtained for each laboratory showed no statistically significant difference among the four laboratories for the overall data set (Table 3) as well as the majority of the individual samples including plasma and the other two low F concentration samples (i.e. RM A $(0.01 \mu \mathrm{gF} / \mathrm{ml})$ and RM B $(0.02 \mu \mathrm{gF} / \mathrm{ml}))$. This method also demonstrated an excellent between-laboratory ICC that ranged from 0.95 to 1.00 with a low $\mathrm{CV}$ ranging between 0.091 to $0.198 \%$ as well as an excellent within-laboratory ICC (0.99-1.00) and low CV (0.022-0.047).

Generally, the sensitivity of the ISE is limited by the Nernstian response slope per decade activity, which is almost $57 \mathrm{mV}$ for the F-ISE. Therefore, the limit of sensitivity of the F-ISE is about 0.02 $\mu \mathrm{g} / \mathrm{ml}(=1 \mu \mathrm{mol} / \mathrm{l})$ which is at, or below, the expected $\mathrm{F}$ concentrations of human plasma samples. However, by using the HMDS diffusion process, the F of the original sample is quantitatively transferred to a smaller volume with higher F concentration; well above the limit of sensitively of the F-ISE [Whitford, 1996]. A recent study [Wang et al., 2017] suggested a universal ion detection method using an electronic integrated multi-electrode system (EIMES) that bypasses the Nernstian slope limit. The EIMES was specified to increase the Nernstian response slope from $57.3 \mathrm{mV}$ for a single F electrode to $564.7 \mathrm{mV}$ for EIMES-10 F electrodes which consequently could record a miniscule change in the ion concentration, improving the accuracy and precision of the measurements. 
The current study found an overall poor between-laboratory ICC $(0.44)$ and CV (1.162) for the microdiffusion method when used to measure micro-litre volumes, indicating a low reliability and high variability with this method. The within-laboratory variation in the overall measured $\mathrm{F}$ concentrations using the micro-diffusion method revealed a wide range in ICC and CVs. The observed betweenlaboratory differences in the present study are consistent with those of Martinez-Mier et al [MartínezMier et al., 2011], who compared the F concentration of several biological samples reported by nine laboratories. Differences in measured F concertation of samples between the laboratories have also been reported in a study that compared $\mathrm{F}$ concentration of water samples analysed by nine laboratories and the laboratory at the South African Bureau of Standards in light of the implementation of water fluoridation in South Africa [Mthethwa and du Plessis, 2005].

Despite the overall poor performance of the micro-diffusion method in the current study, a low variability $(\mathrm{CV}, 0.044)$ and excellent reliability $(\mathrm{ICC}, 1.00)$ was found for Laboratory 4 where that method was developed and is routinely in use. These results highlight the importance of the experience held by the laboratories and its impact on the precision and accuracy of the analytical method. These results confirm that the use of a protocol for $\mathrm{F}$ analysis of samples using the microdiffusion method, without participating in a training programme prior to adoption, might not yield accurate measurement of $\mathrm{F}$ in low volume plasma samples.

When using the known-addition technique in the current study, the overall within-laboratory variation was very low (CVs ranging from 0.030 to 0.068 ) and the reliability was excellent (ICCs ranging from 0.99 to 1.00 ). However, comparison of the results for each individual sample among laboratories indicated statistically significantly differences in F measurements, mainly for those samples with higher concentrations of $\mathrm{F}$, among all four laboratories.

The study results confirmed the established HMDS-diffusion method as the most accurate method for low volume plasma samples. Although the known-addition technique performed better than the micro-diffusion method, it showed a tendency of underestimating F concentration of samples with higher F concentrations ( $>0.06 \mathrm{mgF} / \mathrm{l})$. At a F concentration of $0.02 \mu \mathrm{g} / \mathrm{ml}$, the performance of the known-addition technique was similar to the established HMDS-diffusion method (Figure 1) which was validated by the QQ-plots (Figure 2). The QQ-plots for the HMDS-diffusion method and knownaddition technique were straight, implying good agreement, whereas the micro-diffusion method showed a distinct $\mathrm{S}$-shaped curve suggesting it is not a reliable method for $\mathrm{F}$ measurements of low $\mathrm{F}$ concentration samples. The non-significant difference in $\mathrm{F}$ concentration between the plasma samples measured by the established gold standard method (i.e. HMDS-diffusion) and the known-addition technique, along with the narrow CI, also support the reliability of the known-addition technique for measurement of $\mathrm{F}$ in plasma samples. 
In the current study, a $200 \mu \mathrm{l}$ plasma sample was used for $\mathrm{F}$ determination and the $\mathrm{F}$ analysis of the plasma samples analysed in Phase 3 showed a F concentration of more than $0.020 \mu \mathrm{g} / \mathrm{ml}(=20 \mathrm{ng} / \mathrm{ml})$ for all samples. A satisfactory reproducibility with $150 \mu 1$ plasma samples containing $\geq 20 \mathrm{ng} F$ has also been reported by Ekstrand [Ekstrand, 1977]. However, Ekstrand also noted that for samples containing <20ng F, a minimum volume of $500 \mu \mathrm{l}$ plasma was required for $\mathrm{F}$ determination with reasonable accuracy.

A simple and easy sampling technique, such as capillary sampling, is required for systematic determination of plasma F concentrations, particularly in children. A maximum blood volume of $19 \mu 1$ has been reported for 8-20 year olds when the blood samples were collected by capillary punctures using soft-touch finger pricking devices [Pacaud et al., 1996]. As a result, the amount of blood collected by capillary sampling might be insufficient for accurate and reliable F measurements.

In conclusion, the findings of this study suggest that the known-addition technique could be an alternative option for measurement of $\mathrm{F}$ in plasma with micro-litre volume $(200 \mu \mathrm{l})$ samples. Although the method gives a satisfactory reliability with samples containing $0.01-0.06 \mu \mathrm{gF} / \mathrm{ml}$, above a concentration of $0.06 \mu \mathrm{gF} / \mathrm{ml}$, the method tends to underestimate F concentration of samples. More studies are needed to refine this method or develop other simple methods for $\mathrm{F}$ analysis in micro-litre volume plasma samples containing nano- or sub-nano-gram amounts of F. 


\section{References}

Becker D, Christensen R, Currie L, Diamondstone B, Eberhardt KR, Gills T, Hertz H, Klouda G, Moody J, Parris R, Schaffer R, Steel E, Taylor J, Watters R, Zeisler R: Use of NIST Standard

Reference Materials for Decisions on Performance of Analytical Chemical Methods and Laboratories, Gaithersburg, NIST Special Publication 829, 1992.

Buzalaf MA: Fluoride and the Oral Environment. Basel, Karger, 2011.

Ekstrand J: A micromethod for the determination of fluoride in blood plasma and saliva. Calcif Tissue Res 1977;23:225-228.

Hallsworth AS, Weatherell JA, Deutsch D: Determination of Subnanogram Amounts of Fluoride with Fluoride Electrode. Anal Chem 1976;48:1660-1664.

Martinez-Mier E, Buckley C, Chandrappa P, Soto-Rojas AE: Development of a Standard Fluoride Analytical Method for Dental Plaque. J Dent Res 2010;89:931.

Martínez-Mier EA, Cury J.A., Heilman J.R. , Katz B.P., Levy S.M., Li Y., Maguire A., Margineda J., O’Mullane D., Phantumvanit P., Soto-Rojas A.E., Stookey G.K., Villa A., Wefel J.S., Whelton H. , Whitford G.M., Zero D.T., Zhang W., Zohouri V: Development of gold standard ion-selective electrode-based methods for fluoride analysis Caries Res 2011;45:3-12.

Mthethwa MT, du Plessis JB: A comparison of results of fluoride determinations by different laboratories. SADJ 2005;60:238-240.

Pacaud D, Lemay JF, Guay P, Buithieu M, Yale JF: Assessment of blood volumes obtained by capillary punctures in older children and adolescents with diabetes. Pediatr Res 1996;39:558-558.

PALL Medical: Plasma Optimization Guide: Improving Plasma Yields from Whole Blood Donations. Washington; USA, Pall Corporation. , 2010.

Pinheiro J BD, DebRoy S, Sarkar D and R Core Team: nlme: Linear and Nonlinear Mixed Effects Models. R package version 3.1-131. https://CRAN.R-project.org/package=nlme, 2017.

Pinheiro JC, Bates DM: Mixed effects models in S and S-plus, Springer Verlag, 2000.

Rugg-Gunn AJ, Villa AE, Buzalaf MRA: Contemporary biological markers of exposure to fluoride: Monogr Oral Sci. 2011, vol 22, pp 37-51. 
Taves D: Separation of fluoride by rapid diffusion using hexamethyldisiloxane. Talanata 1968;15:969-974.

Venkateswarlu P: Evaluation of analytical methods for fluorine in biological and related materials. J Dent Res 1990;69 Spec No:514-521; discussion 556-517.

Vogel GL, Carey CM, Chow LC, Ekstrand J: Fluoride analysis in nanoliter- and microliter-size fluid samples. J Dent Res 1990;69:522-528; discussion 556-527.

Wang C, Yuan H, Duan Z, Xiao D: Integrated multi-ISE arrays with improved sensitivity, accuracy and precision. Sci Rep 2017;7:44771.

Whitford GM: The Metabolism and Toxicity of Fluoride. Basel, Karger, 1996.

World Health Organization: Fluorides and oral health. Report of a WHO Expert Committee on Oral Health Status and Fluoride Use. Geneva, Switzerland, 1994. 


\section{Table headings:}

Table 1. Between- and within-laboratory variability for pooled mean (SD) F concentration $(\mu \mathrm{g} / \mathrm{ml})$, combined across all laboratories, by method of analysis. Intra-class Correlation Coefficient (ICC); Coefficient of Variation $(\mathrm{CV})$

Table 2.Mean (SD) and Within-laboratory variability in the overall $\mathrm{F}$ concentration $(\mu \mathrm{g} / \mathrm{ml})$ according to analytical method

Table 3. Comparison of the results for each individual sample* among laboratories by method

Table 4. Comparison of the measured $\mathrm{F}$ concentrations with the true (expected) F concentration values using the mixed-model analysis.

\section{Figure legends:}

Figure 1.Comparison of overall measured and true expected $\mathrm{F}$ concentration by analytical method across all laboratories (red line shows the true expected values)

A: Gold standard HMDS-diffusion method; B: Micro-diffusion method; C: Known-addition Technique

Figure 2. The QQ-plots for gold standard HMDS-diffusion method (A), micro-diffusion method (B) and known-addition Technique (C) for the samples RM-A $(0.01 \mu \mathrm{gF} / \mathrm{ml}), \mathrm{RM}-\mathrm{B}(0.02 \mu \mathrm{gF} / \mathrm{ml})$ and RM-C $(0.06 \mu \mathrm{gF} / \mathrm{ml})$ across all laboratories. 
Table 1. Between- and within-laboratory variability for pooled mean (SD) F concentration $(\mu \mathrm{g} / \mathrm{ml})$, combined across all laboratories, by method of analysis. Intra-class Correlation Coefficient (ICC); Coefficient of Variation (CV)

\begin{tabular}{cccc}
\hline & HMDS-diffusion & Micro-diffusion & Known-addition \\
\hline Between-laboratory & & & \\
Mean (SD) & $0.367(0.047)$ & $0.324(0.376)$ & $0.277(0.135)$ \\
ICC (95\% CI) & $0.99(0.95-1.00)$ & $0.44(0.05-0.77)$ & $0.83(0.54-0.94)$ \\
CV (95\% CI) & $0.129(0.091-0.198)$ & $1.162(0.633-\infty)$ & $0.487(0.325-0.853)$ \\
Within-laboratory & & & \\
Mean (SD) & $0.367(0.011)$ & $0.324(0.036)$ & $0.277(0.013)$ \\
ICC (95\% CI) & $1.00(0.99-1.00)$ & $0.97(0.86-0.99)$ & $1.00(0.98-1.00)$ \\
CV (95\% CI) & $0.031(0.022-0.047)$ & $0.111(0.078-0.169)$ & $0.047(0.033-0.072)$ \\
\hline
\end{tabular}


Table 2.Mean (SD) and Within-laboratory variability in the overall F concentration $(\mu \mathrm{g} / \mathrm{ml})$ according to analytical method

\begin{tabular}{|c|c|c|c|c|c|c|c|c|c|}
\hline \multirow{3}{*}{ Lab } & \multicolumn{9}{|c|}{ Method } \\
\hline & \multicolumn{3}{|c|}{ HMDS-diffusion } & \multicolumn{3}{|c|}{ Micro-diffusion } & \multicolumn{3}{|c|}{ Known-addition } \\
\hline & $\begin{array}{c}\text { Mean } \\
\text { (SD) }\end{array}$ & $\begin{array}{c}\text { ICC } \\
(95 \% \mathrm{CI})\end{array}$ & $\begin{array}{c}\text { CV } \\
(95 \% \mathrm{CI})\end{array}$ & $\begin{array}{l}\text { Mean } \\
\text { (SD) }\end{array}$ & $\begin{array}{c}\text { ICC } \\
(95 \% \mathrm{CI})\end{array}$ & $\begin{array}{c}\text { CV } \\
(95 \% \mathrm{CI})\end{array}$ & $\begin{array}{l}\text { Mean } \\
\text { (SD) }\end{array}$ & $\begin{array}{c}\text { ICC } \\
(95 \% \mathrm{CI})\end{array}$ & $\begin{array}{c}\text { CV } \\
(95 \% \mathrm{CI})\end{array}$ \\
\hline 1 & $\begin{array}{c}0.358 \\
(0.035)\end{array}$ & $\begin{array}{c}0.96 \\
(0.82-0.99)\end{array}$ & $\begin{array}{c}0.097 \\
(0.068-0.148)\end{array}$ & $\begin{array}{c}0.072 \\
(0.048)\end{array}$ & $\begin{array}{c}0.37 \\
(0.00-0.81)\end{array}$ & $\begin{array}{c}0.671 \\
(0.428-1.406)\end{array}$ & $\begin{array}{c}0.249 \\
(0.007)\end{array}$ & $\begin{array}{c}1.00 \\
(0.99-1.00)\end{array}$ & $\begin{array}{c}0.030 \\
(0.021-0.045)\end{array}$ \\
\hline 2 & $\begin{array}{c}0.381 \\
(0.007)\end{array}$ & $\begin{array}{c}1.00 \\
(1.00-1.00)\end{array}$ & $\begin{array}{c}0.018 \\
(0.012-0.027)\end{array}$ & $\begin{array}{c}0.239 \\
(0.041)\end{array}$ & $\begin{array}{c}0.89 \\
(0.59-0.97)\end{array}$ & $\begin{array}{c}0.171 \\
(0.120-0.263)\end{array}$ & $\begin{array}{c}0.322 \\
(0.016)\end{array}$ & $\begin{array}{c}1.00 \\
(0.98-1.00)\end{array}$ & $\begin{array}{c}0.049 \\
(0.035-0.075)\end{array}$ \\
\hline 3 & $\begin{array}{c}0.368 \\
(0.015)\end{array}$ & $\begin{array}{c}1.00 \\
(0.99-1.00)\end{array}$ & $\begin{array}{c}0.040 \\
(0.028-0.060)\end{array}$ & $\begin{array}{c}0.629 \\
(0.059)\end{array}$ & $\begin{array}{c}0.84 \\
(0.44-0.96)\end{array}$ & $\begin{array}{c}0.093 \\
(0.066-0.142)\end{array}$ & $\begin{array}{c}0.371 \\
(0.025)\end{array}$ & $\begin{array}{c}0.99 \\
(0.96-1.00)\end{array}$ & $\begin{array}{c}0.068 \\
(0.048-0.103)\end{array}$ \\
\hline 4 & $\begin{array}{c}0.358 \\
(0.008)\end{array}$ & $\begin{array}{c}1.00 \\
(1.00-1.00)\end{array}$ & $\begin{array}{c}0.023 \\
(0.016-0.035)\end{array}$ & $\begin{array}{c}0.355 \\
(0.016)\end{array}$ & $\begin{array}{c}1.00 \\
(0.98-1.00)\end{array}$ & $\begin{array}{c}0.044 \\
(0.031-0.067)\end{array}$ & $\begin{array}{c}0.165 \\
(0.009)\end{array}$ & $\begin{array}{c}1.00 \\
(0.98-1.00)\end{array}$ & $\begin{array}{c}0.053 \\
(0.037-0.081)\end{array}$ \\
\hline
\end{tabular}


Table 3. Comparison of the results for each individual sample* among laboratories by method

\begin{tabular}{|c|c|c|c|c|}
\hline Method & Sample & Laboratory & $\begin{array}{c}\text { Mean (SE) } \\
\begin{array}{c}\text { Between-laboratory } \\
\text { difference }\end{array} \\
\end{array}$ & $P$ value \\
\hline HMDS-diffusion & RM-C & 2 vs. 4 & $-0.028(0.002)$ & 0.04 \\
\hline \multirow{6}{*}{ Micro-diffusion } & \multirow{2}{*}{ NIST-D } & 1 vs. 3 & $-2.162(0.146)$ & 0.04 \\
\hline & & 1 vs. 4 & $-1.011(0.075)$ & 0.05 \\
\hline & \multirow{2}{*}{ Plasma-2 } & 1 vs. 3 & $-0.019(0.001)$ & 0.02 \\
\hline & & 1 vs. 4 & $-0.012(0.001)$ & 0.02 \\
\hline & \multirow{2}{*}{ UCRF-2 } & 1 vs. 3 & $-0.417(0.033)$ & 0.04 \\
\hline & & 2 vs. 3 & $-0.274(0.022)$ & 0.04 \\
\hline \multirow{19}{*}{ Known-addition } & \multirow{6}{*}{ NIST-A } & 1 vs. 2 & $-0.125(0.007)$ & 0.005 \\
\hline & & 1 vs. 3 & $-0.181(0.007)$ & 0.002 \\
\hline & & 1 vs. 4 & $0.106(0.008)$ & 0.01 \\
\hline & & 2 vs. 3 & $-0.056(0.008)$ & 0.04 \\
\hline & & 2 vs. 4 & $0.231(0.009)$ & 0.002 \\
\hline & & 3 vs. 4 & $0.287(0.009)$ & 0.001 \\
\hline & \multirow{2}{*}{ NIST-B } & 1 vs. 4 & $0.231(0.013)$ & 0.04 \\
\hline & & 2 vs. 4 & $0.446(0.025)$ & 0.04 \\
\hline & \multirow{5}{*}{ NIST-C } & 1 vs. 2 & $-0.127(0.009)$ & 0.03 \\
\hline & & 1 vs. 3 & $-0.178(0.017)$ & 0.05 \\
\hline & & 1 vs. 4 & $0.115(0.007)$ & 0.03 \\
\hline & & 2 vs. 4 & $0.242(0.008)$ & 0.01 \\
\hline & & 3 vs. 4 & $0.293(0.017)$ & 0.03 \\
\hline & \multirow{2}{*}{ NIST-D } & 1 vs. 4 & $0.253(0.016)$ & 0.04 \\
\hline & & 2 vs. 4 & $0.462(0.020)$ & 0.03 \\
\hline & Plasma-3 & 2 vs. 4 & $0.018(0.001)$ & 0.03 \\
\hline & RM-E & 3 vs. 4 & $0.636(0.047)$ & 0.04 \\
\hline & \multirow{2}{*}{ UCRF-2 } & 1 vs. 3 & $-0.094(0.007)$ & 0.04 \\
\hline & & 1 vs. 4 & $0.060(0.006)$ & 0.05 \\
\hline
\end{tabular}

\footnotetext{
* The samples with no statistically significant between-laboratory differences in their measured $\mathrm{F}$ concentration are not presented
} 
Table 4. Comparison of the measured F concentrations with the true (expected) F concentration values using the mixed-model analysis.

\begin{tabular}{|l|l|l|}
\hline Variable & Statistic & P- value \\
\hline Intercept & $\mathrm{F} 1,158=89.18$ & $<0.0001$ \\
\hline True Expected F concentration & $\mathrm{F} 1,158=221.85$ & $<0.0001$ \\
\hline Method & $\mathrm{F} 2,158=0.26$ & 0.7727 \\
\hline Laboratory & $\mathrm{F} 3,158=9.01$ & $<0.0001$ \\
\hline
\end{tabular}




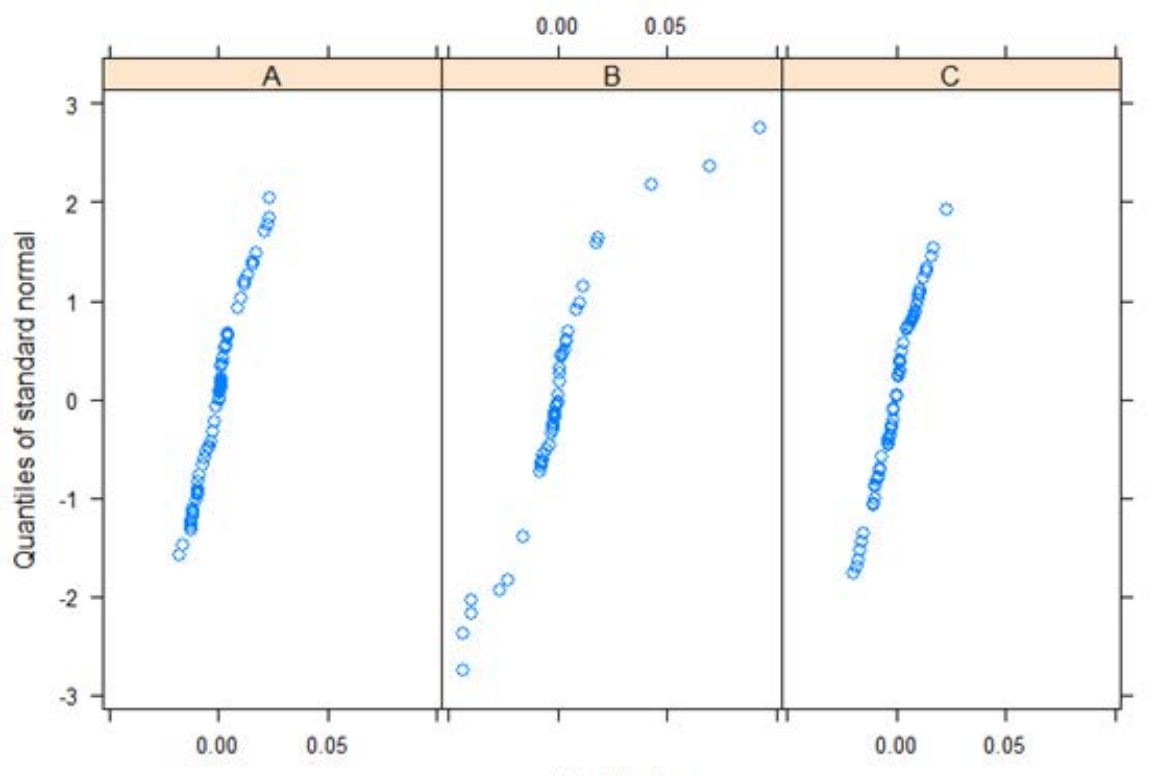

Residuals 